\title{
Discounting by Intervals: A Generalized Model of Intertemporal Choice
}

\author{
Marc Scholten \\ Department of Social and Organizational Psychology, Instituto Superior de Psicologia Aplicada, \\ Rua Jardim do Tabaco 34, 1149-041 Lisboa, Portugal, scholten@ispa.pt \\ Daniel Read \\ Durham Business School, Mill Hill Lane, Durham DH1 3LB, United Kingdom, daniel.read2@durham.ac.uk
}

\begin{abstract}
A ccording to most models of intertemporal choice, an agent's discount rate is a function of how far the outcomes are removed from the present, and nothing else. This view has been challenged by recent studies, which show that discount rates tend to be higher the closer the outcomes are to one another (subadditive discounting) and that this can give rise to intransitive intertemporal choice. We develop and test a generalized model of intertemporal choice, the Discounting By Intervals (DBI) model, according to which the discount rate is a function of both how far outcomes are removed from the present and how far the outcomes are removed from one another. The model addresses past challenges to other models, most of which it includes as special cases, as well as the new challenges presented in this paper: Our studies show that when the interval between outcomes is very short, discount rate tends to increase with interval length (superadditive discounting). In the discussion we place our model and evidence in a broader theoretical context.
\end{abstract}

Key words: intertemporal choice; delay effort; subadditivity; superadditivity; intransitivity

History: Accepted by Detlof von Winterfeldt, decision analysis; received May 8, 2005. This paper did not require revision.

The theory of finance provides decision makers with formal rules that prescribe how intertemporal choices should be made. The basis of these rules is that agents should discount future cash flows by a specific amount that is a function of their risk and the time until they occur (e.g., Brealey and Myers 2002). These rules are usually instantiated in a computer program, so that the process of valuation is taken out of the agents' hands. Their personal time preferences, such as whether they are patient or impatient, do not influence the prescribed value of each option.

Such mechanical valuation does not describe how most intertemporal choices are made, whether in business or personal life. Even momentous choices, such as those concerning career options, pension plans, and even capital expenditures, are routinely made on the basis of intuition. To understand how such choices are made, researchers have studied them empirically. A vast amount of evidence from these studies shows that unaided intertemporal choices differ systematically from those prescribed by the normative principles of finance and economics (for a review, see Frederick et al. 2003). Many researchers, therefore, have developed descriptive models of intertemporal choice. Currently, the most comprehensive description of how real decision makers value and discount future outcomes is provided by Loewenstein and Prelec's (1992) model of intertemporal choice. However, evidence has emerged from a series of recent studies that challenges the descriptive accuracy of this model.

In this paper, we develop and test a generalized model of intertemporal choice, the Discounting By Intervals (DBI) model. While earlier models hold that an agent's discount rate is only a function of how far outcomes are removed from the present, the DBI model suggests that it is also a function of how far they are removed from one another. We show that the DBI model addresses past challenges to Loewenstein and Prelec's model, as well as the new challenges presented in this paper.

\section{Discounting by Delays}

We focus on choices between smaller-sooner (SS) and larger-later $(L L)$ outcomes, such as that between $£ 100$ in one month and $£ 250$ in 13 months. We denote the outcomes as $x_{S}$ and $x_{L}$ ( $£ 100$ and $\left.£ 250\right)$, and their respective delays as $t_{S}$ and $t_{L}$ (one and 13 months). In discounting models, these choices are governed by the discounted values of the options, meaning the values of $x_{S}$ and $x_{L}$ discounted as a function of their 
respective delays. These discounted values are given as

$$
\begin{aligned}
& V\left(x_{S}, t_{S}\right)=d\left(t_{S}\right) v\left(x_{S}\right) \\
& V\left(x_{L}, t_{L}\right)=d\left(t_{L}\right) v\left(x_{L}\right),
\end{aligned}
$$

where $V(x, t)$ is the present value of $x$, or its value given that it will be received after a wait of $t ; v(x)$ is the value $x$ will have when it is received; and $d(t)$ is a discount factor decreasing in $t$. The point at which $S S$ and $L L$ have equal present values, i.e., $d\left(t_{S}\right) v\left(x_{S}\right)=$ $d\left(t_{L}\right) v\left(x_{L}\right)$, is the indifference point. Given the indifference point, we can derive the discount fraction, which is a measure of discounting over the interval $t_{S} \rightarrow t_{L}$ :

$$
F_{t_{S} \rightarrow t_{L}}=\frac{d\left(t_{L}\right)}{d\left(t_{S}\right)}=\frac{v\left(x_{S}\right)}{v\left(x_{L}\right)} .
$$

A higher value of $F$ indicates less discounting. In turn, we can derive a one-period discount fraction, which is a measure of average discounting over the interval:

$$
\delta_{t_{S} \rightarrow t_{L}}=\left[F_{t_{S} \rightarrow t_{L}}\right]^{1 /\left(t_{L}-t_{S}\right)},
$$

where $t_{S}$ and $t_{L}$ are specified in an appropriate unit, usually in years. A higher value of $\delta$ indicates less discounting per unit of time. To illustrate, suppose that an agent is indifferent between ( $£ 100$, one month) and (£250, 13 months), and also between (£100, one month) and (£400, 25 months). If it is assumed that $v(x)=x$, the discount fractions would be $F_{1 \rightarrow 13}=$ $100 / 250=0.4$ and $F_{1 \rightarrow 25}=100 / 400=0.25$, indicating that there is more discounting over the longer interval, while the one-period discount fractions would be $\delta_{1 \rightarrow 13}=(0.4)^{1 / 1}=0.4$ and $\delta_{1 \rightarrow 25}=(0.25)^{1 / 2}=0.5$, indicating that there is less discounting per unit of time over the longer interval.
As described by Equation (1), decision makers take into account only how far outcomes are removed from the present. Thus, SS is discounted over the delay $0 \rightarrow t_{S}$, while $L L$ is discounted over the delay $0 \rightarrow t_{L}$. Accordingly, the discounting over the interval $t_{S} \rightarrow t_{L}$ is derived from the discounting over two delays, as described by Equation (2). It is shown below that this discounting framework is problematic and has to be replaced by a more general one.

\section{The Effects of Delay and Interval}

In the present and the subsequent section, we discuss the four discounting models presented in Table 1 . The first three models, which have been proposed elsewhere, are special cases of the DBI model.

The implications of the discounting models will be discussed in relation to Figure 1, which displays the discount fractions for the interval $t_{S} \rightarrow t_{L}$ and for two subintervals of equal length, $t_{S} \rightarrow t_{M}$ and $t_{M} \rightarrow t_{L}$. To illustrate, an agent could be asked what amount $\left(x_{L}\right)$ received in three years $\left(t_{L}\right)$ would be equal in value to $£ 100\left(x_{S}\right)$ received in one year $\left(t_{S}\right)$. Alternatively, the agent could be asked what amount $\left(x_{M}\right)$ in two years $\left(t_{M}\right)$ would be equally valued to $£ 100$ in one year, and then be asked what amount in three years would be equally valued to $x_{M}$ in two years. The tasks represent a stylized experiment addressing the effect of the delay to interval onset (comparing $F_{t_{S} \rightarrow t_{M}}$ with $F_{t_{M} \rightarrow t_{L}}$ ) and the effect of interval length (comparing $F_{t_{S} \rightarrow t_{M} \rightarrow t_{L}}$ with $F_{t_{S} \rightarrow t_{L}}$ ) on intertemporal preferences.

The first row of Table 1 depicts the exponential discounting model introduced by Samuelson (1937). It is

\begin{tabular}{|c|c|c|c|}
\hline Source & Discount fraction & Domain & Implications \\
\hline Samuelson (1937) & $F_{t_{S} \rightarrow t_{L}}=\left[\frac{e^{t_{S}}}{e^{t_{L}}}\right]^{\beta}=\left[\frac{1}{e^{\left(t_{L}-t_{S}\right)}}\right]^{\beta}$ & $\beta>0$ & Discounting by delays \\
\hline Loewenstein and Prelec (1992) ${ }^{\mathrm{a}}$ & $F_{t_{S} \rightarrow t_{L}}=\left[\frac{1+\alpha t_{S}}{1+\alpha t_{L}}\right]^{\beta / \alpha}=\left[\frac{1+\alpha t_{S}}{1+\alpha t_{S}+\alpha\left(t_{L}-t_{S}\right)}\right.$ & $\begin{array}{l}\beta>0 \\
\alpha>0\end{array}$ & $\begin{array}{l}\text { Discounting by delays } \\
\text { Diminishing sensitivity }\end{array}$ \\
\hline $\operatorname{Read}(2001)^{b}$ & $F_{t_{S} \rightarrow t_{L}}=\left[\frac{1}{e^{\left(t_{L}-t_{S}\right)^{\vartheta}}}\right]^{\beta}$ & $\begin{array}{c}\beta>0 \\
0<\vartheta<1\end{array}$ & $\begin{array}{l}\text { Discounting by intervals } \\
\text { Subadditivity }\end{array}$ \\
\hline DBI model ${ }^{c}$ & $F_{t_{S} \rightarrow t_{L}}=\left[\frac{1}{1+\alpha\left(t_{L}^{\tau}-t_{S}^{\tau}\right)^{\vartheta}}\right]^{\beta / \alpha}$ & $\begin{aligned} \beta & >0 \\
\alpha & >0 \\
\vartheta & >1 \\
0 & <\tau<1\end{aligned}$ & $\begin{array}{l}\text { Discounting by intervals } \\
\text { Subadditivity } \\
\text { Superadditivity } \\
\text { Diminishing sensitivity }\end{array}$ \\
\hline
\end{tabular}
the benchmark for other models because it can be derived from relatively uncontroversial choice axioms

Table 1 Four Discounting Models: Discount Fraction, Parameter Domain, and Implications

aReduces to Samuelson's (1937) exponential discounting model when $\alpha \downarrow 0$ and to Harvey's (1986) and Mazur's (1987) hyperbolic discounting models when $\alpha=1$ and $\alpha=\beta$, respectively. It is equivalent to Rachlin's (1989) hyperbolic discounting model, in which $\beta / \alpha=s$.

${ }^{\mathrm{b}}$ Reduces to Samuelson's (1937) exponential discounting model when $\vartheta=1$.

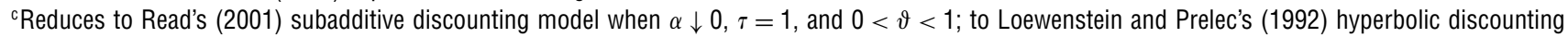
model when $t_{S}=0$ and $\tau=\vartheta=1$; and to Mazur's (1987) exponentiated hyperbolic discounting model when $t_{S}=0$ (so that $\tau$ is absorbed into $\vartheta$ ) and $\alpha=\beta$. 
Figure 1 An Undivided Interval and an Interval Divided into Two Subintervals

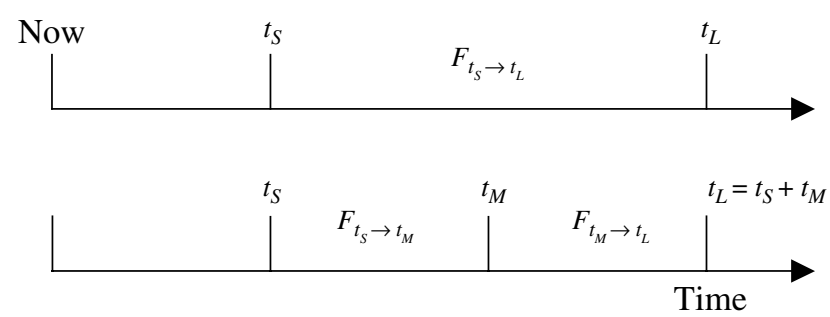

(Koopmans 1960, Lancaster 1963) and because it is applied in most economic analyses of intertemporal choice (Loewenstein and Prelec 1992). According to the exponential discounting model, the discount fraction is stationary over intervals of equal length, i.e., $F_{S \rightarrow M}=F_{M \rightarrow L}$. Stationarity has long been viewed as a poor characterization of intertemporal choice, in that there tends to be more discounting over an interval the closer it is to the present (Samuelson 1937, Strotz 1955-1956). A typical example was reported by Keren and Roelofsma (1995). They found that a majority preferred $S S$ when choosing between

$$
\begin{aligned}
& A=(f 100, \text { now }) \text { and } \\
& B=(f 110, \text { four weeks }),
\end{aligned}
$$

but a majority preferred $L L$ when choosing between

$$
\begin{aligned}
C & =(f 100,26 \text { weeks }) \text { and } \\
D & =(f 110,30 \text { weeks }) .{ }^{1}
\end{aligned}
$$

This choice pattern suggests that there is more discounting over an interval of four weeks that begins immediately than over one that begins in 26 weeks, or, more generally, that $F_{S \rightarrow M}<F_{M \rightarrow L}$. This effect of the delay to interval onset has been called the delay effect (Thaler 1981).

Most descriptive models of intertemporal choice capture the delay effect, in that the discount fraction for an interval increases as the delay to the onset of the interval increases (e.g., Harvey 1986, Loewenstein and Prelec 1992, Mazur 1987, Rachlin 1989). The second row of Table 1 depicts the hyperbolic discounting model proposed by Loewenstein and Prelec (1992), which, with few exceptions, includes other discounting models as special cases. ${ }^{2}$ The formula is expanded in the table to show that because $t_{S}$ is in both the numerator and the denominator, the discount fraction

\footnotetext{
${ }^{1}$ The outcomes were Dutch guilders $(f)$, which at the time were about a third of British pounds.

${ }^{2}$ The major exception is the quasi-hyperbolic discounting, or present-biased preference, model explored by Laibson (1997) and O'Donoghue and Rabin (1999). This model assumes a single jolt of discounting for any delay, but exponential discounting thereafter. It thus captures an immediacy effect rather than a general delay effect.
}

for the interval $t_{S} \rightarrow t_{L}$ increases as the delay to interval onset, $0 \rightarrow t_{S}$, increases.

The delay effect is a manifestation of what is known as diminishing sensitivity in the analysis of decision under risk and uncertainty: Marginal impact diminishes with distance from a reference point (Tversky and Fox 1995, Tversky and Kahneman 1992). For outcomes, the natural reference point is the status quo, so that the difference between $£ 110$ and $£ 100$ has less impact than that between $£ 10$ and nothing. For delays, the natural reference point is the present, so that the difference between 30 weeks and 26 weeks has less impact than that between four weeks and now, which is the delay effect.

Exponential and hyperbolic discounting models differ as to whether the delay to interval onset will have an effect on intertemporal preferences, but agree that interval length will have no effect whatsoever. The reason is that these models assume that discounting over an interval is derived from discounting over two delays. As described by Equation (2), the discount fraction for interval $t_{S} \rightarrow t_{L}$ is equal to the ratio between the factor by which $L L$ is discounted over the delay $0 \rightarrow t_{L}$ and the factor by which $S S$ is discounted over the delay $0 \rightarrow t_{S}$. Thus, in relation to Figure 1, these models predict that the discounting over the divided interval $t_{S} \rightarrow t_{M} \rightarrow t_{L}$ will be equal to the discounting over the undivided interval $t_{S} \rightarrow t_{L}$ :

$$
\begin{aligned}
F_{t_{S} \rightarrow t_{M} \rightarrow t_{L}} & =F_{t_{S} \rightarrow t_{M}} \times F_{t_{M} \rightarrow t_{L}}=\frac{d\left(t_{M}\right)}{d\left(t_{S}\right)} \times \frac{d\left(t_{L}\right)}{d\left(t_{M}\right)} \\
& =\frac{d\left(t_{L}\right)}{d\left(t_{S}\right)}=F_{t_{S} \rightarrow t_{L}} .
\end{aligned}
$$

More generally, exponential and hyperbolic discounting models predict that the discount fraction will be unaffected by dividing an interval into subintervals.

This prediction, however, has not received any empirical support. Rather, all available evidence suggests that there is more discounting over an interval that is divided into subintervals than over an interval that is left undivided, i.e., $F_{t_{S} \rightarrow t_{M} \rightarrow t_{L}}<F_{t_{S} \rightarrow t_{L}}$ (Baron 2000, Read 2001, Read and Roelofsma 2003). ${ }^{3}$ This

\footnotetext{
${ }^{3}$ This effect of interval length is probably responsible for the robust result that, when holding the delay to interval onset constant, there is more discounting per unit of time over a longer interval than over a shorter one. While purportedly substantiating the delay effect, this result actually demonstrates the effect of interval length, albeit confounded with the effect of the delay to the larger-later outcome (see also Read 2001). The delay effect itself, i.e., the effect of the delay to interval onset, has been surprisingly elusive. It has emerged in some studies (Green et al. 1994, Keren and Roelofsma 1995, Kirby and Herrnstein 1995) but not, or not reliably, in others (Ahlbrecht and Weber 1997, Baron 2000, Holcomb and Nelson 1992, Read 2001, Read and Roelofsma 2003). Moreover, the positive results actually demonstrate an immediacy effect, rather than a general delay effect (see also Footnote 2). The effect of interval length, however, has emerged in every relevant study.
} 
effect of interval length has been called subadditive discounting. ${ }^{4}$

Earlier we illustrated the delay effect with a choice pattern where $S S$ is chosen when an interval begins immediately, but $L L$ is chosen when it begins after a delay. We now illustrate subadditive discounting with a choice pattern where $S S$ is chosen when an interval is divided into subintervals, but $L L$ is chosen when it is left undivided. The example is taken from Roelofsma and Read's (2000) study of intransitive intertemporal choice. Consider the following options:

$$
\begin{aligned}
A & =(f 7,1 \text { week }), \\
B & =(f 8,2 \text { weeks }), \\
C & =(f 9,4 \text { weeks }), \\
D & =(f 10,7 \text { weeks }) .
\end{aligned}
$$

The modal choice cycle was preference for $S S$ when choosing from adjacent pairs $(A \succ B, B \succ C$, and $C \succ D$ ), but preference for $L L$ when choosing from the most distant pair $(A \prec D)$, revealing a subadditive weighing of intervals.

The third row of Table 1 depicts the subadditive discounting model that Read (2001) proposed to describe his experimental results, which showed an effect of interval length but no effect of the delay to interval onset. In relation to Figure 1, this model predicts that the discount fraction for the divided interval $t_{S} \rightarrow$ $t_{M} \rightarrow t_{L}$ will be lower (indicating more discounting) than the discount fraction for the undivided interval $t_{S} \rightarrow t_{L}$. For instance, if $\beta=1$,

$$
\begin{aligned}
F_{t_{S} \rightarrow t_{M} \rightarrow t_{L}} & =F_{t_{S} \rightarrow t_{M}} \times F_{t_{M} \rightarrow t_{L}}=\frac{1}{e^{\left(t_{M}-t_{S}\right)^{\vartheta}}} \times \frac{1}{e^{\left(t_{L}-t_{M}\right)^{\vartheta}}} \\
& =\frac{1}{e^{\left(t_{M}-t_{S}\right)^{\vartheta}+\left(t_{L}-t_{M}\right)^{\vartheta}}}<\frac{1}{e^{\left(t_{L}-t_{S}\right)^{\vartheta}}}=F_{t_{S} \rightarrow t_{L}}
\end{aligned}
$$

provided that $0<\vartheta<1$. Thus, the sum (the undivided interval) has less weight than its parts (the subintervals), i.e., $\left(t_{L}-t_{S}\right)^{\vartheta}<\left(t_{M}-t_{S}\right)^{\vartheta}+\left(t_{L}-t_{M}\right)^{\vartheta}$, underscoring the subadditivity in intervals.

\footnotetext{
${ }^{4}$ Subadditive discounting refers to subadditivity in intervals. Hyperbolic discounting, as described by Loewenstein and Prelec's (1992) model in Table 1, gives rise to subadditivity in delays. In our earlier example of diminishing sensitivity to delays, the difference between 30 weeks and 26 weeks has less impact than that between four weeks and now. Under the assumption that discounting is only a function of the delay to outcomes, the example suggests that $d(4) / d(0)<d(30) / d(26)$. Immediate outcomes are not discounted, i.e., $d(0)=1$, so that, under Loewenstein and Prelec's formulation of the discount function, $\log (1+\alpha 30)<\log (1+\alpha 26)+\log (1+\alpha 4)$. Thus the logarithmic function $w(t)=\log (1+\alpha t)$ describes a subadditive weighing of delays, analogous to a subadditive weighing of probabilities in decision under risk and uncertainty (Tversky and Fox 1995). In this paper, however, subadditivity refers specifically to subadditivity in intervals.
}

The subadditive discounting model, while capturing the effect of interval length, raises several problems. First, the model lacks a theoretical basis: It offers a formula for the discounting over an interval without justifying whether and how it can be accommodated by a discounting framework. For instance, it is incompatible with the framework in Equation (1). Second, the model fails to capture the delay effect, the main focus of most other discounting models. Third, subadditive discounting is not the only effect of interval length. We show below that it can reverse into superadditive discounting when an interval is divided into short subintervals.

\section{Discounting by Intervals}

In this section, we develop the DBI model, which suggests that decision makers take into account both how far outcomes are removed from the present and how far they are removed from one another. According to our model, $S S$ is discounted over the interval $0 \rightarrow t_{S}$, while $L L$ is discounted over the consecutive intervals $0 \rightarrow t_{S}$ and $t_{S} \rightarrow t_{L} \cdot{ }^{5}$ As a result, discounting over the interval $t_{S} \rightarrow t_{L}$ has a primitive, rather than derivative, status. In the DBI model, the discounted values are given as

$$
\begin{aligned}
& V\left(x_{S}, t_{S}\right)=D\left(0, t_{S}\right) v\left(x_{S}\right) \\
& V\left(x_{L}, t_{L}\right)=D\left(0, t_{S}\right) D\left(t_{S}, t_{L}\right) v\left(x_{L}\right),
\end{aligned}
$$

where $D$ is a discount function with two arguments: the moment when an interval begins and the moment when it ends. Indifference between $S S$ and $L L$ arises when

$$
D\left(0, t_{S}\right) v\left(x_{S}\right)=D\left(0, t_{S}\right) D\left(t_{S}, t_{L}\right) v\left(x_{L}\right)
$$

or, because the factors by which the outcomes are discounted over the interval $0 \rightarrow t_{S}$ cancel out,

$$
v\left(x_{S}\right)=D\left(t_{S}, t_{L}\right) v\left(x_{L}\right) .
$$

Thus, according to the DBI model, the discount fraction for the interval $t_{S} \rightarrow t_{L}$ is equal to the factor by which $L L$ is discounted over the interval $t_{S} \rightarrow t_{L}$ :

$$
F_{t_{S} \rightarrow t_{L}}=D\left(t_{S}, t_{L}\right)=\frac{v\left(x_{S}\right)}{v\left(x_{L}\right)} .
$$

Note that this is a generalization of Equation (2), which imposes the restriction that

$$
D\left(t_{S}, t_{L}\right)=\frac{d\left(t_{L}\right)}{d\left(t_{S}\right)}
$$

\footnotetext{
${ }^{5}$ While the time segment $0 \rightarrow t_{S}$ may be viewed either as a delay or as an interval, the DBI model treats all time segments as intervals, regardless of whether they begin in the present or in the future. However, it is convenient to preserve our terminological distinction between the interval $t_{S} \rightarrow t_{L}$ and the delay to interval onset, $0 \rightarrow t_{S}$.
} 
The subadditive discounting model proposed by Read (2001) is compatible with the generalized discounting framework in Equation (4), which resolves the first problem raised by this model. The second problem can be addressed by generalizing the subadditive discounting model itself. As mentioned earlier, the delay effect is a manifestation of diminishing sensitivity. This notion, which is widely used in the analysis of decision under risk, has its roots in the WeberFechner law, which relates physical stimulus intensity to subjective magnitude through a logarithmic function (see also Footnote 4), and to its successor Stevens's law, which relates physical stimulus intensity to subjective magnitude through a power function. In the analysis of decision under risk and uncertainty, a concave power function is used to capture diminishing sensitivity to outcomes (e.g., Tversky and Kahneman 1992):

$$
v(x)=x^{\gamma},
$$

where $0<\gamma<1$. Analogously, a concave power function may be used to capture diminishing sensitivity to delays:

$$
w(t)=t^{\tau},
$$

where $0<\tau<1$. Thus, the subadditive discounting model captures the delay effect when expanded by the weighing function in Equation (9), resolving the second problem raised by this model.

The third problem is that subadditive discounting is not the only effect of interval length. Specifically, as we show in two experiments below, there is superadditive discounting when an interval is divided into short subintervals. The impetus for these experiments were two studies of decision under risk, showing that intransitive risky choice may result from a subadditive weighing of probability differences when outcome increments are small relative to probability increments (Leland 1994) or from a superadditive weighing of probability differences when probability increments are small relative to outcome increments (Tversky 1969). To illustrate the latter situation, participants in Tversky's study were presented with all possible pairs from the following set of risky prospects:

$$
\begin{aligned}
A & =(\$ 5.00,7 / 24), \\
B & =(\$ 4.75,8 / 24), \\
C & =(\$ 4.50,9 / 24), \\
D & =(\$ 4.25,10 / 24), \\
E & =(\$ 4.00,11 / 24) .
\end{aligned}
$$

A common choice cycle was preference for the prospect with the greater prize when choosing from adjacent pairs $(A \succ B, B \succ C, C \succ D$, and $D \succ E)$, but preference for the prospect with the greater probability of winning when choosing from the most distant pair $(A \prec D)$, revealing a superadditive weighing of probability differences.

Our suggestion is that either form of nonadditivity can also occur in intertemporal choice. Roelofsma and Read (2000), whose study of intransitive intertemporal choice was discussed in the previous section, investigated a situation where outcome increments were small relative to time increments, thus obtaining subadditivity in time differences (intervals). Therefore, we investigate a situation where time increments are small relative to outcome increments, expecting to obtain superadditivity in intervals. Moreover, we examine whether superadditivity changes into subadditivity when shorter intervals are progressively combined into longer ones. Such a change from one form of nonadditivity in attribute differences to another has never been demonstrated before.

The progressive change from superadditivity to subadditivity can be addressed by further generalizing the subadditive discounting model. According to this generalized model, when expanded by the weighing function in Equation (9), the discount fraction for the interval $t_{S} \rightarrow t_{L}$ is

$$
F_{t_{S} \rightarrow t_{L}}=\left[\frac{1}{e^{\left(t_{L}^{\tau}-t_{S}^{\tau}\right)^{\vartheta}}}\right]^{\beta}
$$

where $\beta>0$ (discounting by intervals), $0<\tau<1$ (diminishing sensitivity to delays), and $0<\vartheta<1$ (subadditivity in intervals). However, our suggestion is that discounting is not always subadditive in intervals, but that it reverses into superadditive discounting when the interval $t_{S} \rightarrow t_{L}$ is perceived to be short. The DBI model, as developed in the last row of Table 1, describes such a discounting pattern. The formula is a generalization of Equation (10), which imposes the restriction that

$$
F_{t_{S} \rightarrow t_{L}}=\lim _{\alpha \downarrow 0}\left[\frac{1}{1+\alpha\left(t_{L}^{\tau}-t_{S}^{\tau}\right)^{\vartheta}}\right]^{\beta / \alpha}
$$

The DBI model includes two nonadditivity parameters, $\alpha$ and $\vartheta$. The $\alpha$-parameter is the one that vanishes in the subadditive discounting model, i.e., $\alpha \downarrow 0$, but not in the DBI model, i.e., $\alpha>0$. The $\alpha$ parameter produces diminishing sensitivity to intervals (i.e., subadditivity) in the DBI model, just as it produces diminishing sensitivity to delays in Loewenstein and Prelec's (1992) model. Thus, in relation to Figure 1, the DBI model predicts that the discount fraction for the divided interval $t_{S} \rightarrow t_{M} \rightarrow t_{L}$ will usually be lower 
Figure 2 Discount Fractions and One-Period Discount Fractions for Different Interval Lengths and Different Delays to Interval Onset

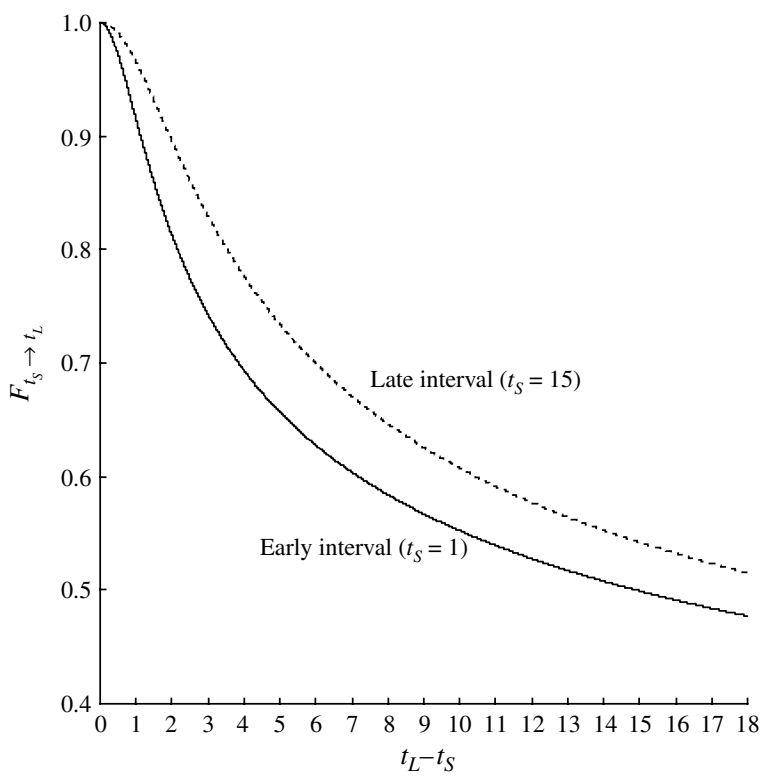

than the discount fraction for the undivided interval $t_{S} \rightarrow t_{L}$. For instance, if $\alpha=\beta=\tau=\vartheta=1$,

$$
\begin{aligned}
F_{t_{S} \rightarrow t_{M} \rightarrow t_{L}} & =F_{t_{S} \rightarrow t_{M}} \times F_{t_{M} \rightarrow t_{L}} \\
& =\frac{1}{1+\left(t_{M}-t_{S}\right)} \times \frac{1}{1+\left(t_{L}-t_{M}\right)} \\
& =\frac{1}{1+\left(t_{L}-t_{S}\right)+\left(t_{M}-t_{S}\right)\left(t_{L}-t_{M}\right)} \\
& <\frac{1}{1+\left(t_{L}-t_{S}\right)}=F_{t_{S} \rightarrow t_{L}} .
\end{aligned}
$$

Discounting will usually be subadditive, but not always. The $\vartheta$-parameter, which produces subadditivity in the subadditive discounting model, i.e., $0<$ $\vartheta<1$, produces superadditivity in the DBI model, i.e., $\vartheta>1$, thus countervailing the influence of the $\alpha$-parameter. To illustrate, if the $\alpha$-parameter does not exercise its influence, i.e., $\alpha \downarrow 0$, and if $\beta=\tau=1$,

$$
\begin{aligned}
F_{t_{S} \rightarrow t_{M} \rightarrow t_{L}} & =F_{t_{S} \rightarrow t_{M}} \times F_{t_{M} \rightarrow t_{L}}=\frac{1}{e^{\left(t_{M}-t_{S}\right)^{\vartheta}}} \times \frac{1}{e^{\left(t_{L}-t_{M}\right)^{\vartheta}}} \\
& =\frac{1}{e^{\left[\left(t_{M}-t_{S}\right)^{\vartheta}+\left(t_{L}-t_{M}\right)^{\vartheta}\right]}}>\frac{1}{e^{\left(t_{L}-t_{S}\right)^{\vartheta}}}=F_{t_{S} \rightarrow t_{L}}
\end{aligned}
$$

provided that $\vartheta>1 .^{6}$ If both parameters exercise their influence, $\alpha$ and $\vartheta$ produce a pattern of superadditive and subadditive discounting over intervals of increasing length.

Figure 2 illustrates how, according to the DBI model, discount fractions, $F_{t_{S} \rightarrow t_{L}}$, and one-period

\footnotetext{
${ }^{6}$ Past applications of an exponentiated hyperbolic discount function over raw delays supported the conclusion that $\vartheta>1$ (e.g., Mazur 1987, Rodriguez and Logue 1988).
}

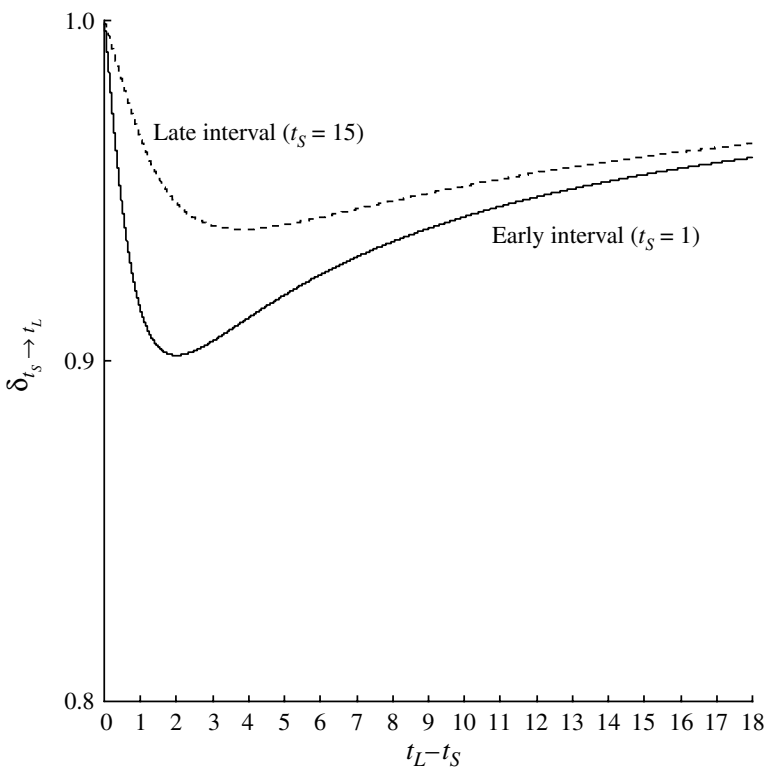

discount fractions, $\delta_{t_{S} \rightarrow t_{L}}$, are affected by the delay to interval onset and interval length. As can be seen, $F_{t_{s} \rightarrow t_{L}}$ and $\delta_{t_{S} \rightarrow t_{L}}$ are higher the more interval onset is delayed (the delay effect). Moreover, as interval length increases, $\delta_{t_{S} \rightarrow t_{L}}$ first decreases (superadditivity) and then increases (subadditivity).

Below we report two experiments that document both superadditivity and subadditivity in the discounting of future outcomes. Because superadditivity has not previously been demonstrated in the domain of intertemporal choice, it was the focus of Experiment 1. We obtain a choice cycle where $L L$ is preferred when intervals are short, but $S S$ is preferred when short intervals are combined into longer ones, revealing superadditivity in intervals. ${ }^{7}$ Experiment 2 is a choice-titration study showing that as shorter intervals are progressively combined into longer ones, $\delta_{t_{S} \rightarrow t_{L}}$ first decreases (superadditivity) and then increases (subadditivity). Experiment 2 also allows us to estimate the parameters of the DBI model.

\section{Experiment 1}

Experiment 1 is a choice study, examining whether discounting over short intervals is more likely to be

\footnotetext{
${ }^{7}$ We will provide a "clean" demonstration of intransitive intertemporal choice by presenting participants only with information about delays and outcomes (cf. Roelofsma and Read 2000). This is in contrast with past demonstrations of intransitive risky choice, where choice cycles were promoted by visual displays: Blackedout, pie-shaped areas on otherwise white disks blurred small differences along the probability attribute (Tversky 1969) or the money attribute (Leland 1994), but not larger differences.
} 
Table 2 Stimuli of Choice Study: Option Pairs and Compound Interest Rates

\begin{tabular}{ccc}
\hline \multicolumn{2}{c}{ Option pairs } & \\
\cline { 1 - 2 }$S S$ & \multicolumn{1}{c}{$L L$} & $r_{t_{S} \rightarrow t_{L}}{ }^{\mathrm{a}, \mathrm{b}}$ \\
\hline$A=£ 500$ in 1 week & $B=£ 525$ in 2 weeks & 0.0500 \\
$B=£ 525$ in 2 weeks & $C=£ 550$ in 3 weeks & 0.0476 \\
$C=£ 550$ in 3 weeks & $D=£ 575$ in 4 weeks & 0.0455 \\
$A=£ 500$ in 1 week & $C=£ 550$ in 3 weeks & 0.0488 \\
$B=£ 525$ in 2 weeks & $D=£ 575$ in 4 weeks & 0.0465 \\
$A=£ 500$ in 1 week & $D=£ 575$ in 4 weeks & 0.0477 \\
\hline${ }^{\mathrm{a}} r_{t_{S} \rightarrow t_{L}}=\left(x_{L} / x_{S}\right)^{1 /\left(t_{L}-t_{S}\right)}-1$. & \\
${ }^{\mathrm{b} A l l}$ compound interest rates round to $5 \%$ per week.
\end{tabular}

superadditive than subadditive. The length of each subinterval was one week, and the compensation for waiting was $£ 25$ (British pounds) per week, in the expectation that three waits of one week for three compensations of $£ 25$ would be less painful than one wait of three weeks for one compensation of $£ 75$ (superadditive discounting), so that participants would choose $L L$ over the subintervals, but $S S$ over the undivided interval. Table 2 exhibits the six option pairs used in this study, together with the compound interest rate for each pair.

\section{Method}

A random order of the six option pairs was determined and a Latin square of six different orders was designed on the basis of that random order. Six different questionnaires were prepared on the basis of the Latin square. The questionnaires presented each option pair on a separate page, in the following format:

\begin{tabular}{lcc}
\hline & Option 1 & Option 2 \\
\hline You receive: & $£ 500$ & $£ 525$ \\
When: & 1 week from today & 2 weeks from today \\
Your choice: & $\square$ & $\square$
\end{tabular}

The participants, 120 students from the London School of Economics, checked the box corresponding to the option they preferred.

\section{Results}

Given all possible dyadic choices between four options, there are 64 possible choice patterns. We can divide these, following the method of Roelofsma and Read (2000), into 24 transitive patterns (T-patterns) and 40 intransitive ones (I-patterns). An I-pattern is one that contains at least one choice cycle. To illustrate the difference, consider the following four preference relations between $A, B$, and $C$ :

$$
\begin{array}{rr}
B \succ A, \quad C \succ B, \quad \text { and } \quad C \succ A \\
\quad \text { (T-pattern, willing to wait); } \\
B \succ A, \quad C \succ B, \quad \text { but } A \succ C \\
\quad \text { (I-pattern, superadditivity); } \\
A \succ B, \quad B \succ C, \quad \text { and } A \succ C \\
\quad \text { (T-pattern, unwilling to wait); } \\
A \succ B, \quad B \succ C, \quad \text { but } C \succ A \\
\quad \text { (I-pattern, subadditivity). }
\end{array}
$$

Out of the 120 questionnaires, fully 84 were T-patterns, primarily because most participants restricted their choices to $S S$ or $L L$ alone. We focus on the I-patterns, because only those patterns can reveal superadditivity and subadditivity.

The I-patterns can be divided into those that exhibit (i) superadditivity, (ii) both superadditivity and a delay effect, (iii) subadditivity, (iv) both subadditivity and a delay effect, and (v) an anomalous pattern. A delay effect is observed when SS is preferred to $L L$ over an early interval, but $L L$ is preferred to $S S$ over a later interval of the same length. An example would be when $A \succ B$, but $C \succ B .^{8}$ Subadditivity is observed when, as in the example above, $S S$ is preferred to $L L$ over the subintervals, but $L L$ is preferred to SS over the undivided interval. Superadditivity is observed when, as in the example above, $L L$ is preferred to $S S$ over the subintervals, but $S S$ is preferred to $L L$ over the undivided interval. The anomalous I-patterns are those that violate the delay effect, i.e., when $L L$ is preferred to $S S$ over an early interval, but $S S$ is preferred to $L L$ over a later interval of the same length. Table 3 provides a classification of all nonanomalous I-patterns (those in categories (i) to (iv)) and the frequency of their occurrence.

The occurrence rates of superadditivity and subadditivity were 12 and five, respectively, so superadditive patterns were more than twice as frequent as subadditive ones. However, we should take into account that the base rate is different for superadditivity, which can be revealed by nine choice patterns; and subadditivity, which can be revealed by only seven choice patterns. Moreover, we should take into account that choice behavior has error associated with it, so that a participant may have "accidentally"

\footnotetext{
${ }^{8}$ As can be seen in Table 2, interest rates are slightly higher over early intervals than over later ones of the same length, so that the delay effect (by which more compensation is demanded over the former than over the latter) can be observed only if it outweighs the decline in interest rates.
} 
Scholten and Read: Discounting by Intervals

Table 3 Results of Choice Study: Nonanomalous I-Patterns and Frequency of Occurrence

\begin{tabular}{|c|c|c|c|c|c|c|c|c|c|c|}
\hline \multirow{3}{*}{$\begin{array}{l}\text { Delay } \\
\text { effect }\end{array}$} & \multicolumn{5}{|c|}{ Superadditivity } & \multicolumn{5}{|c|}{ Subadditivity } \\
\hline & \multirow[b]{2}{*}{ Option } & \multicolumn{3}{|c|}{ Option } & \multirow[b]{2}{*}{$N$} & \multirow[b]{2}{*}{ Option } & \multicolumn{3}{|c|}{ Option } & \\
\hline & & $B$ & $C$ & $D$ & & & $B$ & $C$ & $D$ & $\Lambda$ \\
\hline \multirow[t]{18}{*}{ Yes } & $A$ & $L L$ & $S S$ & $L L$ & & $A$ & $S S$ & $L L$ & $L L$ & \\
\hline & $B$ & & $L L$ & $L L$ & & $B$ & & SS & $L L$ & \\
\hline & $C$ & & & $L L$ & 0 & $C$ & & & $L L$ & 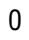 \\
\hline & $A$ & $L L$ & SS & SS & & $A$ & SS & SS & $L L$ & \\
\hline & $B$ & & $L L$ & $L L$ & & $B$ & & SS & $S S$ & \\
\hline & $C$ & & & $L L$ & 0 & $C$ & & & $L L$ & 0 \\
\hline & $A$ & SS & $L L$ & SS & & $A$ & SS & SS & $L L$ & \\
\hline & $B$ & & $L L$ & $L L$ & & $B$ & & SS & $L L$ & \\
\hline & C & & & $L L$ & 2 & $C$ & & & SS & \\
\hline & $A$ & $S S$ & $S S$ & $L L$ & & $A$ & SS & $S S$ & SS & \\
\hline & $B$ & & $L L$ & $S S$ & & $B$ & & $S S$ & $L L$ & \\
\hline & C & & & $L L$ & 0 & C & & & SS & c \\
\hline & $A$ & SS & SS & SS & & & & & & \\
\hline & $B$ & & $L L$ & SS & & & & & & \\
\hline & $C$ & & & $L L$ & 5 & & & & & \\
\hline & $A$ & SS & $L L$ & SS & & & & & & \\
\hline & $B$ & & $S S$ & $L L$ & & & & & & \\
\hline & $C$ & & & $L L$ & 0 & & & & & \\
\hline \multirow[t]{9}{*}{ No } & $A$ & $L L$ & $L L$ & SS & & $A$ & SS & $L L$ & $L L$ & \\
\hline & $B$ & & $L L$ & $L L$ & & $B$ & & $S S$ & $L L$ & \\
\hline & C & & & $L L$ & 2 & C & & & SS & 2 \\
\hline & $A$ & $L L$ & SS & $L L$ & & $A$ & $S S$ & $L L$ & SS & \\
\hline & $B$ & & $L L$ & SS & & $B$ & & $S S$ & $L L$ & \\
\hline & $c$ & & & $L L$ & 1 & C & & & SS & 0 \\
\hline & $A$ & $L L$ & SS & SS & & $A$ & SS & $S S$ & $L L$ & \\
\hline & $B$ & & $L L$ & SS & & $B$ & & SS & $S S$ & \\
\hline & $C$ & & & $L L$ & 2 & $C$ & & & $S S$ & \\
\hline Total & & & & & 12 & & & & & 5 \\
\hline
\end{tabular}

Note. A delay effect is observed when there is a shift from $S S$ to $L L$ when moving down on one or both diagonals. Intransitivity can be detected by looking at "triangles" of responses - two or three diagonal items and a pivot item on the same row as the first of these items and the same column as the last (e.g., the diagonal items $B C$ and $C D$, and the pivot item $B D$ ). Superadditivity or subadditivity is observed when all diagonal items differ from the pivot item.

ended up in the superadditive category, the subadditive category, or indeed the anomalous category. We therefore relate the frequency with which each of these categories occurs to the frequency with which it can be expected to occur by chance alone. Specifically, for each category, we calculate:

Expected frequency

$$
\begin{aligned}
= & \frac{\text { Number of choice patterns in category }}{\text { Total number of choice patterns }} \\
& \times \text { Number of participants. }
\end{aligned}
$$

Table 4 presents the number of choice patterns, the expected and observed frequency, and the $\chi^{2}$-deviation between the expected and observed frequency for each category. As can be seen, anomalous I-patterns occurred less often than expected,
Table 4 Results of Choice Study: Category Statistics for I-Patterns

\begin{tabular}{lcccc}
\hline & \multicolumn{4}{c}{ Choice pattern } \\
\cline { 2 - 4 } Category statistic & Superadditivity & Subadditivity & Anomalous & Total \\
\hline No. of choice patterns & 9 & 7 & 24 & 40 \\
Expected frequency & 6.75 & 5.25 & 18 & 30 \\
Observed frequency & 12 & 5 & 13 & 30 \\
$\chi^{2}$-deviation & 4.08 & 0.01 & 1.39 & 5.48 \\
\hline${ }^{a} \chi^{2}$-deviation $=($ Expected frequency & - Observed frequency $)^{2} /$ Expected
\end{tabular}
frequency.

subadditivity occurred as often as expected, while superadditivity occurred more often than expected. This result was significant, $\chi^{2}(2)=5.48, p=0.06$.

Experiment 1 has demonstrated that there exists a range of interval lengths over which discounting is superadditive. Experiment 2 integrates this result with subadditive discounting, in that, over a wider range of interval lengths, discounting is first superadditive and then subadditive.

\section{Experiment 2}

Experiment 2 is a choice-titration study, examining whether discounting is superadditive over short intervals but subadditive over longer ones. A choicetitration study, which analyzes indifference relations, should be more diagnostic than a choice study, which analyzes preference relations. For instance, if, across a series of option pairs, $v\left(x_{S}\right)<D\left(t_{S}, t_{L}\right) v\left(x_{L}\right)$, LL will always be chosen over $S S$, regardless of whether the discount function $d$ is nonadditive over intervals. However, if repeated choices are made from each option pair and the delay or outcome of one option is adjusted following each choice until the point is reached where $v\left(x_{S}\right)=D\left(t_{S}, t_{L}\right) v\left(x_{L}\right)$, the nonadditivity of the discount function can be exposed. Moreover, this also allows us to estimate the parameters in the proposed discount function.

In this study, repeated choices were made between option pairs, $S S$ and $L L$, with the outcome of one option, $x_{S}$ or $x_{L}$, being adjusted following each choice, until participants were close to the point of indifference between the options. The choice-titration procedure is described in the online appendix available at http://mansci.pubs.informs.org/ecompanion.html.

There were nine option pairs, corresponding to intervals of three different lengths: Six short intervals of one week, two medium-length intervals of three weeks, and one long interval of 17 weeks. There was a medium-length interval at the beginning and end of the long interval, the earlier one denoted $e, m$; the later one denoted $l, m$; and the long interval denoted l. Each medium-length interval comprised three short intervals, the three constituting the earlier one denoted $e 1, s ; e 2, s$; and $e 3, s$; and the three constituting the later one denoted $l 1, s ; l 2, s$; and $l 3, s$. Given the delays 
and outcomes of the nine option pairs at the point of indifference, we conducted both a qualitative and a quantitative analysis.

Qualitative Analysis. We computed 13 one-period discount fractions. Nine $\delta$ s were obtained for the nine option pairs, applying Equation (3) under the assumption of a linear value function $v$. The other four $\delta$ s were aggregate discount fractions, obtained by computing geometric means, viz.,

$$
\begin{aligned}
& \delta_{\cdot, m}=\left(\delta_{e, m} \times \delta_{l, m}\right)^{1 / 2}, \\
& \delta_{e \cdot, s}=\left(\delta_{e 1, s} \times \delta_{e 2, s} \times \delta_{e 3, s}\right)^{1 / 3}, \\
& \delta_{l, s}=\left(\delta_{l 1, s} \times \delta_{l 2, s} \times \delta_{l 3, s}\right)^{1 / 3}, \text { and } \\
& \delta_{\cdot \cdot, s}=\left(\delta_{e \cdot, s} \times \delta_{l \cdot, s}\right)^{1 / 2} .
\end{aligned}
$$

Our first hypothesis was that $\delta$ would be lower for early intervals than for later intervals of the same length (the delay effect):

Hypothesis 1 (H1). $\delta_{e \cdot, s}<\delta_{l, s,} ; \delta_{e, m}<\delta_{l, m}$.

Our second hypothesis was that $\delta$ would be higher for short intervals than for intervals of medium length (superadditivity), and lower for medium-length intervals than for long ones (subadditivity):

Hypothesis 2 (H2). $\delta_{. \cdot, s}>\delta_{\cdot, m}<\delta_{l}$.

We also tested strong superadditivity and subadditivity, which would occur if interval effects outweighed the delay effect. Consider again Figure 1. By the delay effect alone, $\delta$ will be lower over the early interval than over the later one: $\delta_{t_{S} \rightarrow t_{M}}<\delta_{t_{M} \rightarrow t_{L}}$. Therefore, if two intervals of different length begin at the same time, the delay effect results in a lower $\delta$ for the shorter one: $\delta_{t_{S} \rightarrow t_{M}}<\delta_{t_{S} \rightarrow t_{L}}$. Superadditivity, however, leads to a higher $\delta$ for the shorter interval: $\delta_{t_{S} \rightarrow t_{M}}>$ $\delta_{t_{S} \rightarrow t_{L}}$. Strong superadditivity occurs if superadditivity outweighs the delay effect: For two intervals of different length that begin at the same time, $\delta$ is higher for the shorter interval than for the longer one. Applying this definition to our design,

Hypothesis 2A (H2A). $\delta_{e 1, s}>\delta_{e, m} ; \delta_{l 1, s}>\delta_{l, m}$.

Analogously, if two intervals of different length end at the same time, the delay effect results in a lower $\delta$ for the longer one: $\delta_{t_{S} \rightarrow t_{L}}<\delta_{t_{M} \rightarrow t_{L}}$. Subadditivity, however, leads to a higher $\delta$ for the longer interval: $\delta_{t_{S} \rightarrow t_{L}}>\delta_{t_{M} \rightarrow t_{L}}$. Strong subadditivity occurs if subadditivity outweighs the delay effect: For two intervals of different length that end at the same time, $\delta$ is higher for the longer interval than for the shorter one. Applying this definition to our design,

Hypothesis 2B (H2B). $\delta_{l}>\delta_{l, m}$.
Quantitative Analysis. According to the DBI model, the discount fraction for the interval $t_{S} \rightarrow t_{L}$ is

$$
F_{t_{S} \rightarrow t_{L}}=\left[\frac{1}{1+\alpha\left(t_{L}^{\tau}-t_{S}^{\tau}\right)^{\vartheta}}\right]^{\beta / \alpha}=\frac{v\left(x_{S}\right)}{v\left(x_{L}\right)} \text {. }
$$

While our qualitative analysis assumed a linear value function $v$, the quantitative analysis may consider the concave value function in Equation (8). Given that value function, the discount fraction for the interval $t_{S} \rightarrow t_{L}$ is

$$
F_{t_{S} \rightarrow t_{L}}=\left[\frac{1}{1+\alpha\left(t_{L}^{\tau}-t_{S}^{\tau}\right)^{\vartheta}}\right]^{\beta / \alpha}=\left[\frac{x_{S}}{x_{L}}\right]^{\gamma} .
$$

The problem arises that $\gamma$, the exponent of the value function, can be absorbed into $\beta$, the exponent of the discount function, so that it cannot be estimated. The quantitative analysis, therefore, also assumes a linear value function, acknowledging that $\beta$ may be overestimated. Specifically, we estimated the parameters of a model that predicted the larger outcome for each option pair:

$$
\hat{x}_{L}=x_{S}\left[1+\alpha\left(t_{L}^{\tau}-t_{S}^{\tau}\right)^{\vartheta}\right]^{\beta / \alpha} .
$$

We tested whether the parameters departed significantly from their benchmark values in the expected direction:

Hypothesis 3 (H3). $\beta>0$ (discounting by intervals);

Hypothesis 4A (H4A). $\alpha>0$ (subadditivity);

Hypothesis 4B (H4B). $\vartheta>1$ (superadditivity);

Нyротнеsis 5 (H5). $\tau<1$ (diminishing sensitivity).

\section{Method}

The participants were 53 students from the London School of Economics, who were paid $£ 5 .{ }^{9}$ Experimental sessions were run in a computer room with several participants at a time. The participants were seated at a desk with an IBM-compatible computer. An instruction sheet informed them they would choose between (hypothetical) amounts of money available at different times. The instruction sheet illustrated how the situations would be presented on the computer screen. The participants were asked to attend closely to the

\footnotetext{
${ }^{9}$ The participants were paid a flat fee. Making their payment for participation contingent on their choices, e.g., by honoring the delay and payoff chosen on a randomly selected trial (e.g., Kirby and Herrnstein 1995) might have added some realism to their choices. However, given our intention to induce superadditive discounting over short intervals, the payoffs on all trials were substantial ( $£ 500$ and over), so that contingent payment was unfeasible. Moreover, it has been concluded elsewhere that similar results are obtained with and without contingent payment in experiments involving simple trade-offs between delay and payoff (Chapman et al. 1999) or risk and payoff (Tversky and Kahneman 1992).
} 
amounts of money as well as the amounts of time, because both would frequently change throughout the experimental session. The participants were also instructed how to respond: The left and right arrow keys were used to make choices, the down arrow key confirmed choices, and the up arrow key allowed them to correct mistakes.

Before the experimental session, participants completed a series of practice trials starting with $£ 400$ in 26 weeks and $£ 500$ in 52 weeks. This was followed by 18 series of experimental trials, comprising two replications of the nine option pairs.

\section{Results}

Admission of Participants. The analyses were conducted on the results from 42 participants. The remaining results were not used for two reasons. Firstly, the titration task was designed to elicit a choice of $L L$ over $S S$ on the first trial by any reasonable standard (demanding a simple interest of less than 100\% per week). Four participants nonetheless chose $S S$ over $L L$ on the first trial for at least one interval. Secondly, seven participants did not display weak monotonicity, meaning that, on at least one occasion, they demanded more compensation for a shorter interval than for a longer one when the shorter interval was a subset of the longer one. To illustrate, weak monotonicity would be violated by a participant who was indifferent between $£ 500$ and $£ 750$ over the medium-length interval $1 \rightarrow 4$ weeks, but was indifferent between $£ 500$ and $£ 800$ over the short interval $1 \rightarrow 2$ weeks. The survival rate of $79 \%$ of the sample is typical of those reported in other studies (e.g., 81\% in Ahlbrecht and Weber 1997, 72\% in Benzion et al. 1989, and 84\% in Shelley 1993). Table 5 provides the summary results for the 42 participants admitted to the analyses.

Qualitative Analysis. We report four analyses of variance (ANOVA) with $\log (\delta)$ as dependent variable, testing the four hypotheses formulated earlier. ${ }^{10}$

The Delay Effect. The left panel of Figure 3 displays the geometric means of $\delta$ for early and late, short and medium-length intervals. In support of $\mathrm{H} 1$, $\delta$ was lower for early intervals than for later intervals of the same length, confirming the delay effect. In an ANOVA with interval onset (early versus late) and interval length (short versus medium-length) as within-participant factors, the main effect of interval onset was significant, $F(1,41)=7.28, p=0.01$, $\eta^{2}=0.15$.

In addition to the delay effect, superadditivity manifested itself, in that $\delta$ was higher for short intervals

\footnotetext{
${ }^{10}$ Arithmetic means of $\log (\delta)$ preserve the functional relation between $\delta$ s because, for instance, $\log (0.4)-\log (0.2)=\log (0.8)-$ $\log (0.4)$.
}

Table 5 Choice-Titration Study: Stimuli and Summary Results

\begin{tabular}{|c|c|c|c|c|c|c|}
\hline \multirow[b]{2}{*}{ Interval } & \multicolumn{3}{|c|}{ Stimuli } & \multicolumn{3}{|c|}{ Summary results ${ }^{\mathrm{a}}$} \\
\hline & $t_{S}$ & $t_{L}$ & $x_{S}$ & $\bar{X}_{L}^{b}$ & $\hat{x}_{L}^{c}$ & $\bar{\delta}_{t_{S} \rightarrow t_{L}, \mathrm{~d}, \mathrm{e}}$ \\
\hline$e 1, s$ & 1 & 2 & 500 & 542.36 & 546.52 & 0.92 \\
\hline$e 2, s$ & 2 & 3 & 500 & 529.57 & 538.21 & 0.94 \\
\hline$e 3, s$ & 3 & 4 & 500 & 531.66 & 533.51 & 0.94 \\
\hline$e, m$ & 1 & 4 & 500 & 678.69 & 672.55 & 0.90 \\
\hline$/ 1, s$ & 15 & 16 & 500 & 523.04 & 518.25 & 0.96 \\
\hline $12, s$ & 16 & 17 & 500 & 527.22 & 517.77 & 0.95 \\
\hline $13, s$ & 17 & 18 & 500 & 522.72 & 517.34 & 0.96 \\
\hline$I, m$ & 15 & 18 & 500 & 599.25 & 603.46 & 0.94 \\
\hline I & 1 & 18 & 500 & $1,029.15$ & $1,029.81$ & 0.96 \\
\hline
\end{tabular}

${ }^{\mathrm{e}}$ Geometric means preserve the functional relation between $\delta \mathrm{s}$, whereas arithmetic means would not. To illustrate, $\delta=0.2$ denotes twice the discounting of $\delta=0.4$, which, in turn, denotes twice the discounting of $\delta=0.8$. The average amount of discounting is correctly reflected by the geometric mean $(0.4)$, not by the arithmetic mean $(0.467)$.

than for intervals of medium length. The main effect of interval length was significant, $F(1,41)=6.39, p=$ $0.02, \eta^{2}=0.14$.

Superadditivity and Subadditivity. The right panel of Figure 3 displays the geometric means of $\delta$ for short, medium-length, and long intervals. In support of $\mathrm{H} 2$, $\delta$ was higher for short intervals than for intervals of medium length, and lower for medium-length intervals than for long ones, confirming the U-shaped relation between interval length and one-period discount fractions. In an ANOVA with interval length (short versus medium-length versus long) as a withinparticipant factor, the effect of interval length was significant, $F(2,82)=8.43, p=0.00, \eta^{2}=0.17$. Moreover, this effect was located almost entirely in the quadratic contrast, $F(1,41)=11.23, p=0.00, \eta^{2}=0.22$, confirming more directly the U-shaped relation.

Strong Superadditivity. In support of $\mathrm{H} 2 \mathrm{~A}, \delta$ was higher for a short interval than for an interval of medium length when both began equally early, $\bar{\delta}_{e 1, s}=$ 0.92 and $\bar{\delta}_{e, m}=0.90$, or equally late, $\bar{\delta}_{l 1, s}=0.96$ and $\bar{\delta}_{l, m}=0.94$, confirming strong superadditivity. In an ANOVA with interval length (short versus mediumlength) and interval onset (early versus late) as withinparticipant factors, the main effect of interval length was marginally significant, $F(1,41)=3.58, p=0.07$, $\eta^{2}=0.08$.

While outweighed by superadditivity, the delay effect did manifest itself, in that $\delta$ was lower for early intervals than for later intervals of the same length. The main effect of interval onset was significant, $F(1,41)=6.51, p=0.01, \eta^{2}=0.14$.

Strong Subadditivity. In support of $\mathrm{H} 2 \mathrm{~B}, \delta$ was higher for a long interval than for an interval of 
Figure 3 Results of Choice-Titration Study
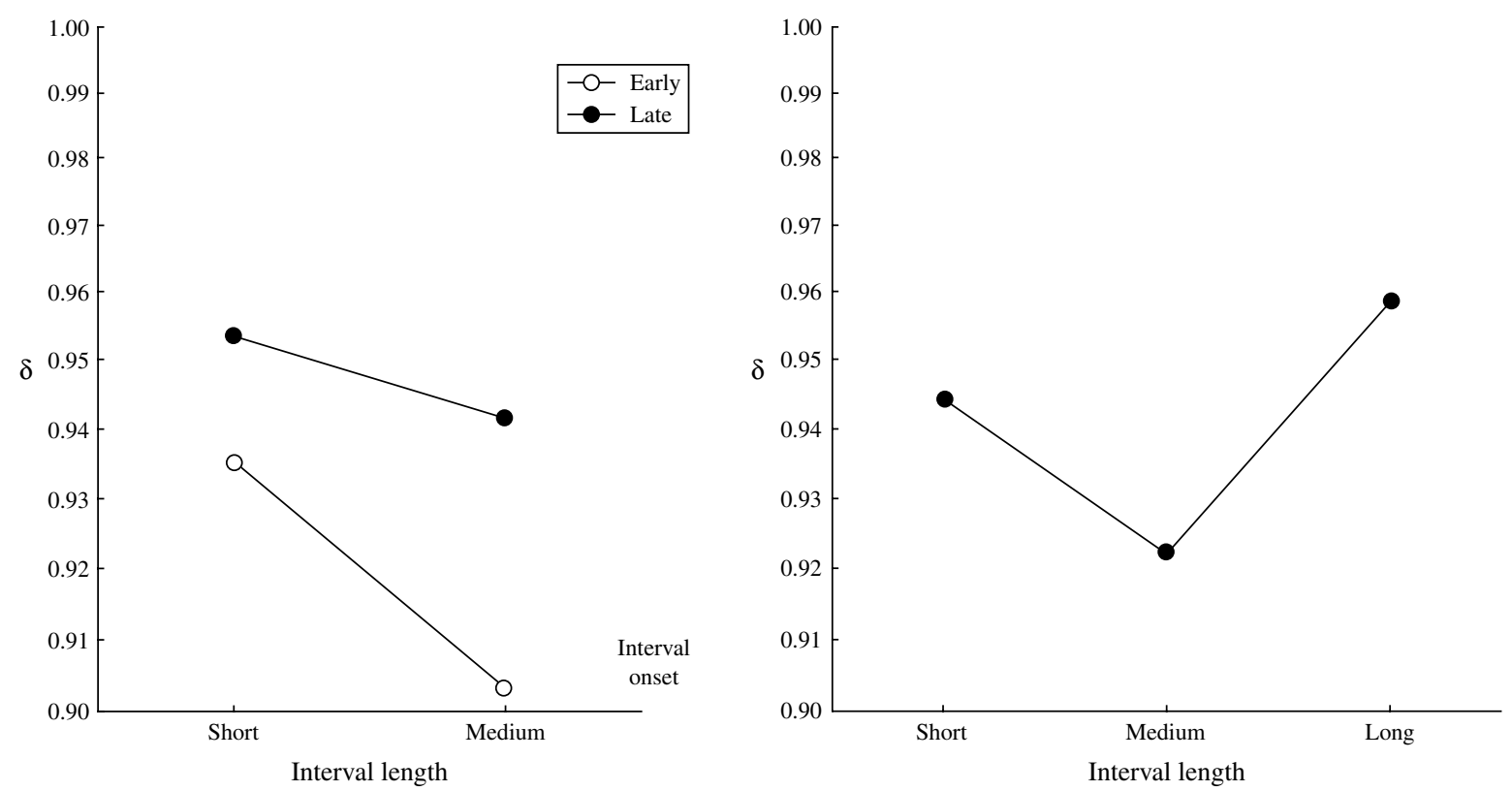

Note. The left panel compares $\delta$ over early and late, short and medium-length intervals, displaying diminishing sensitivity to delays, i.e., $\delta_{e, s}<\delta_{l, s}$ and $\delta_{e, m}<\delta_{l, m}$, and superadditivity in intervals, i.e., $\delta_{e, s}>\delta_{e, m}$ and $\delta_{l, s}>\delta_{l, m}$. The right panel compares $\delta$ over short, medium-length, and long intervals, displaying superadditivity and subadditivity in intervals, i.e., $\delta_{., s}>\delta_{,, m}<\delta_{l}$.

medium length when both ended equally late, $\bar{\delta}_{l}=$ 0.96 and $\bar{\delta}_{l, m}=0.94$, confirming strong subadditivity. In an ANOVA with interval length (medium-length versus long) as a within-participant factor, the effect of interval length was significant, $F(1,41)=4.11, p=$ $0.05, \eta^{2}=0.09$.

Quantitative Analysis. We ran a nonlinear regression, minimizing, with the use of the LevenbergMarquardt search routine of STATISTICA 6, the sum of squared deviations between $\bar{x}_{L}$ and $\hat{x}_{L}$ (see Table 5). As can be seen in Table 6, all parameters departed

Table 6 Choice-Titration Study: Parameter Estimates of the DBI Model

\begin{tabular}{lccc}
\hline \multicolumn{4}{c}{$\begin{array}{c}\text { Dependent variable: } \bar{X}_{L} \\
\text { Goodness-of-fit: } 100 \% \times R^{2}=99.87 \%\end{array}$} \\
\hline Parameter & Estimate & $t(5)^{\mathrm{a}}$ & $p$ \\
\hline$\beta$ & $0.25^{\mathrm{b}}$ & 5.03 & 0.00 \\
$\alpha$ & 1.69 & 3.18 & 0.01 \\
$\vartheta$ & 2.07 & 3.72 & 0.01 \\
$\tau$ & 0.77 & 7.15 & 0.00 \\
\hline
\end{tabular}

${ }^{a}$ Evaluates whether the parameters depart significantly from their benchmark values in the expected direction (one-tailed $t$-tests).

${ }^{b}$ Estimate obtained under the assumption that $\gamma=1$ in Equation (8). We tested the robustness of this result by setting $\gamma<1$, rerunning the regression analysis, and testing whether $\beta$ fell significantly below the estimate of 0.25 . When $\gamma=0.88$ (the estimate of $\gamma$ obtained by Tversky and Kahneman 1992), $\beta$ fell to 0.22 , an insignificant change, $t(4)=-0.73, p=0.25$. When $\gamma=0.77$ (the estimate of $\tau$, as obtained under the assumption that $\gamma=1$ ), $\beta$ fell to 0.19 , once again an insignificant change, $t(4)=-1.55$, $p=0.10$. from their benchmark values in the expected direction. Specifically, the parameters indicated discounting by intervals $(\beta>0)$ in support of H3, subadditivity $(\alpha>$ $0)$ in support of H4A, superadditivity $(\vartheta>1)$ in support of $\mathrm{H} 4 \mathrm{~B}$, and diminishing sensitivity $(\tau<1)$ in support of H5. The functions in Figure 2 were drawn with the parameters estimates in Table 6.

\section{Discussion}

Samuelson's (1937) classic, "A note on measurement of utility," introduced a model of intertemporal choice that belongs to a general class of discounting models described above by Equation (1). In the model formulated by Samuelson, which remains the historical reference point for the normative theory of intertemporal choice, the discount function $d$ is an exponential function defined over raw delays. "For simplicity," Samuelson wrote, "we assume [...] that the rate of discount of future utilities is a constant," adding that this assumption "is in the nature of an hypothesis, subject to refutation by the observable facts" (p. 156). He appeared to know that the exponential discounting model, while mathematically convenient, was unlikely to describe real decision makers. ${ }^{11}$ Psychologists and experimentally minded economists who took up the challenge of understanding what real decision makers did soon discovered that it was anything but discounting at a constant rate. One branch

${ }^{11} \mathrm{He}$ did not even put it forward as a model of rational choice. That interpretation came later. 
of this research, focusing on choices between smallersooner and larger-later outcomes, has revealed that discount rates are highly variable even in this controlled setting. First, as has long been conjecturedeven by Samuelson-discount rates tend to be higher the closer the outcomes are to the present. This "delay effect," which violates the stationarity axiom of normative theory, has been addressed by many descriptive models of intertemporal choice. Second, as discovered more recently, discount rates tend to be higher the closer the outcomes are to one another. This "interval effect" violates the transitivity axiom of normative theory (e.g., Lancaster 1963). Third, as demonstrated for the first time in our experiments, the interval effect reverses when the outcomes are in close range to one another: Then, discount rates tend to be lower again the closer the larger-later outcome is to the smaller-earlier one. To deal with these highly variable discount rates, we have introduced a generalized discounting model, the DBI model, described above by Equation (4). In the DBI model, the discount function $D$ is an exponentiated hyperbolic function defined over perceived intervals. While it sacrifices the convenient simplicity of Samuelson's (1937) model so as to better describe what real decision makers do, we feel that the generalized model is an elegant formulation in spite of its greater complexity.

Future applications of the DBI model may reveal further intricacies. Indeed, in designing our first experiment, we drew on our intuitions about interval lengths and compensations for waiting that would induce superadditive discounting. We felt that a oneweek interval would be sufficiently short relative to a $£ 25$ compensation and that a three-week interval would be sufficiently long relative to a $£ 75$ compensation to induce superadditive discounting. In Roelofsma and Read's (2000) study of intransitive intertemporal choice, discussed earlier, later intervals were slightly longer than earlier ones (one week, two weeks, and three weeks) and the compensations for waiting were much smaller (one-third of $£ 1$ per interval), producing subadditive discounting. In combination, these results suggest that the range of interval lengths over which discounting is superadditive depends on the outcomes being considered. For instance, the discounting over an interval of one week may be superadditive when the compensation for waiting is $£ 25$, but not when it is $£ 1$. Such outcome-dependent discounting violates the separability axiom of normative theory (e.g., Fishburn and Rubinstein 1982). From an applied perspective, this suggests that some parameters of the DBI model may be variable, rather than constant, across contexts involving different compensations but the same intervals. Such parameter variability has many precedents in psychology and economics. For instance, the "psychophysical power law," which relates physical stimulus intensity to subjective magnitude, has a modality-dependent exponent. The different exponents inform us about the nature of the different modalities. For instance, people are decreasingly sensitive to brightness (as they are to delays and outcomes) but increasingly sensitive to heaviness (Stevens 1957). Similarly, applications of the DBI model may show us how discounting varies across contexts involving outcomes of different magnitude or sign.

An online supplement to this paper is available on the Management Science website (http://mansci.pubs. informs.org/ecompanion.html).

\section{Acknowledgments}

The research reported in this paper was funded by the ESRC research grant RES-000-22-0201 and the Leverhulme grant F/07004/Y. Burcu Orsel and Mara Airoldi assisted in the design of the experiments and in the collection of the data.

\section{References}

Ahlbrecht, M., M. Weber. 1997. An empirical study on intertemporal decision making under risk. Management Sci. 43 813-826.

Baron, J. 2000. Can we use human judgments to determine the discount rate? Risk Anal. 20 861-868.

Benzion, U., A. Rapoport, J. Yagil. 1989. Discount rates inferred from decisions: An experimental study. Management Sci. 35 270-284.

Brealey, R. A., S. Myers. 2002. The Principles of Corporate Finance. McGraw-Hill, New York.

Chapman, G. B., R. Nelson, D. B. Hier. 1999. Familiarity and time preferences: Decision making about treatments for migraine headaches and Crohn's disease. J. Experiment. Psych.: Appl. 5 17-34.

Fishburn, P. C., A. Rubinstein. 1982. Time preference. Internat. Econom. Rev. 23 677-694.

Frederick, S., G. Loewenstein, T. O’Donoghue. 2003. Time discounting and time preference: A critical review. G. Loewenstein, D. Read, R. F. Baumeister, eds. Time and Decision. Russell Sage Foundation, New York, 13-86.

Green, L., N. Fristoe, J. Myerson. 1994. Temporal discounting and preference reversals in choice between delayed outcomes. Psychonomic Bull. Rev. 1 383-389.

Harvey, C. M. 1986. Value functions for infinite-period planning. Management Sci. 32 1123-1139.

Holcomb, J. H., P. S. Nelson. 1992. Another experimental look at individual time preference. Rationality Soc. 4 199-220.

Keren, G., P. Roelofsma. 1995. Immediacy and certainty in intertemporal choice. Organ. Behav. Human Decision Process 63 287-297.

Kirby, K. N., R. J. Herrnstein. 1995. Preference reversals due to myopic discounting of delayed reward. Psych. Sci. 6 83-89.

Koopmans, T. C. 1960. Stationary ordinal utility and impatience. Econometrica 28 287-309.

Laibson, D. 1997. Golden eggs and hyperbolic discounting. Quart. J. Econom. 62 443-477.

Lancaster, K. 1963. An axiomatic theory of consumer time preference. Internat. Econom. Rev. 4 221-231.

Leland, J. 1994. Generalized similarity judgments: An alternative explanation for choice anomalies. J. Risk Uncertainty 9 151-172. 
Loewenstein, G., D. Prelec. 1992. Anomalies in intertemporal choice: Evidence and an interpretation. Quart. J. Econom. 107 573-597.

Mazur, J. E. 1987. An adjusting procedure for studying delayed reinforcement. M. L. Commons, J. E. Mazur, L. A. Nevin, H. Rachlin, eds. Quantitative Analyses of Behavior, Vol. 5. Lawrence Erlbaum, Hillsdale, NJ, 55-73.

O'Donoghue, T., M. Rabin. 1999. Doing it now or later. Amer. Econom. Rev. 89 103-124.

Rachlin, H. 1989. Judgment, Decision, and Choice. Freeman, New York.

Read, D. 2001. Is time-discounting hyperbolic or subadditive? J. Risk Uncertainty 23 5-32.

Read, D., P. H. M. P. Roelofsma. 2003. Subadditive versus hyperbolic discounting: A comparison of choice and matching. Organ. Behav. Human Decision Process 91 140-153.

Rodriguez, M. L., A. W. Logue. 1988. Adjusting delay to reinforcement: Comparing choice in pigeons and humans. J. Experiment. Psych.: Animal Behav. Process 14 105-117.
Roelofsma, P. H. M. P., D. Read. 2000. Intransitive intertemporal choice. J. Behav. Decision Making 13 161-177.

Samuelson, P. 1937. A note on measurement of utility. Rev. Econom. Stud. 4 155-161.

Shelley, M. K. 1993. Outcome signs, question frames, and discount rates. Management Sci. 37 770-786.

Stevens, S. S. 1957. On the psychophysical law. Psych. Rev. 64 153-181.

Strotz, R. H. 1955-1956. Myopia and inconsistency in dynamic utility maximization. Rev. Econom. Stud. 23 165-180.

Thaler, R. 1981. Some empirical evidence on dynamic inconsistency. Econom. Lett. 8 201-207.

Tversky, A. 1969. Intransitivity of preferences. Psych. Rev. 76 31-48.

Tversky, A., C. F. Fox. 1995. Weighing risk and uncertainty. Psych. Rev. 102 269-283.

Tversky, A., D. Kahneman. 1992. Advances in prospect theory: Cumulative representation of uncertainty. J. Risk Uncertainty 5 297-323. 